\title{
Greek surgical patients' satisfaction related to perioperative anesthetic services in an academic institute
}

This article was published in the following Dove Press journal:

Patient Preference and Adherence

10 August 2012

Number of times this article has been viewed

\author{
Pinelopi Kouki' \\ Paraskevi Matsota ${ }^{2}$ \\ Kalliopi Christodoulaki \\ Maria Kompoti ${ }^{3}$ \\ Marilia Loizou ${ }^{2}$ \\ Periandros Karamanis ${ }^{2}$ \\ Aggeliki Pandazi ${ }^{2}$ \\ Georgia Kostopanagiotou ${ }^{2}$ \\ 'Department of Anesthesiology, \\ General Hospital, Agios Panteleimon, \\ Nikea, Greece; ${ }^{2}$ Second Department \\ of Anesthesiology, School of Medicine, \\ University of Athens, "Attikon" \\ Hospital, Athens, Greece; 'Intensive \\ Care Unit, Thriassion General \\ Hospital of Eleusis, Athens, Greece
}

Correspondence: Paraskevi Matsota School of Medicine, University of Athens, "Attikon" Hospital, Rimini I Str, Chaidari,

Postal Code 12462,

Athens, Greece

Tel +302105015827

Fax +30210532 6413

Email matsota@yahoo.gr
Background: Patient satisfaction is an increasingly appreciated measure of outcome for health care procedures. The purpose of this study was to evaluate Greek surgical patients' satisfaction with perioperative anesthetic services and to determine which factors maximize satisfaction level through all phases of perioperative care.

Methods: Adult Greek patients admitted for elective surgery in an academic hospital were included in the study. Three separate questionnaires were constructed: Q1 (patients who underwent general anesthesia alone or combined with epidural) and Q2 (patients who received regional anesthesia alone) covered perioperative anesthetic care; Q3 covered postoperative analgesia services in the ward (patient-controlled analgesia or epidural analgesia) provided by our anesthesiologist-centered analgesia care team. Principal component analysis with varimax rotation was used separately for each questionnaire, and extracted factors were entered into multiple logistic regression with patient satisfaction as the dependent binary variable. Statistical significance level was set at $P<0.05$.

Results: Three hundred and forty-five patients were included. Q1 questionnaire (answered by 282 patients) included four dimensions: communication with the anesthesiologist, sense of cold/shivering, pain, and nausea. Q2 questionnaire (answered by 63 patients) included three dimensions: communication with the anesthesiologist, sense of cold/shivering, and nausea/ anxiety. Q3 questionnaire (answered by 237 patients) included five dimensions: anesthesiologist intervention upon symptoms, pain, care by the anesthesiologist/physical activity, nausea/ vomiting, and anesthesiologist behavior. The communication dimension score in Q1 and Q2, sense of shivering in Q2, and pain management and anesthesiologist behavior dimension scores in Q3 were significantly associated with patient satisfaction. Overall satisfaction rates were high (according to the questionnaire, the observed percentage was in the range of 96.3\%-98.6\%).

Conclusion: Greek surgical patients reported high satisfaction with perioperative anesthesia care. Interaction between patient and anesthesiologists during all periods of study, absence of shivering in regional anesthesia, and adequate postoperative pain control in the ward were significant predictors of patient satisfaction in the present Greek surgical population.

Keywords: Greek surgical patients, satisfaction, questionnaire, anesthetic management, postoperative analgesia care team

\section{Introduction}

Recently, recording of patient satisfaction has been considered by the medical community as an important parameter in the analysis of administered health care services. ${ }^{1,2}$ It is considered to be decisive for two reasons: first, it may enable the tracing and correction of health care system impotencies and problems; and second, this approach can meet patients' desires and expectations, resolve their speculations, and consequently, 
increase their trust and cooperation in the national health care system policy and practice. ${ }^{3}$ Factors affecting satisfaction are under investigation throughout the world. Thus far, several validated studies in different ethnicities have been performed regarding anesthesia services. ${ }^{3-7}$

The aim of this study was to evaluate satisfaction in Greek surgical patients regarding perioperative anesthetic care and determine which factors maximize satisfaction level through all phases of the perioperative period. A secondary aim was to search for dissatisfaction points that would reveal our institute's reduced efficiency in our practice. This is the first study, to our knowledge, that measures patient satisfaction in a Greek surgical patient sample regarding anesthesia services. The investigation of different populations with special intrinsic features and dissimilarities further validates the current knowledge of the factors associated with patient satisfaction.

\section{Materials and methods}

After approval from the "Attikon" Hospital Ethics Committee (Athens, Greece), the protocol was instituted and written informed consent from participants was obtained. All Greek adult patients who underwent elective surgery during a threemonth period in our academic hospital were included in this prospective observational study. Exclusion criteria were as follows: age under 16 years, patient refusal, cognitive dysfunction or any other inability to fill the questionnaires on one's own, pregnancy, and postoperative admission in the intensive care unit.

\section{Questionnaire development}

We performed a cross-sectional survey using single-item questions that concerned patient satisfaction with perioperative anesthesia services. According to the existing literature, four basic sections should be investigated: the amount of information given; participation in decision making; the level of physical comfort and presence of side effects; and the presence of emotional support, with respect of patient values. Three pilot questionnaires (based on three valid and psychometrically designed studies) were delivered to 60 participants..$^{3-5}$ Pilot questionnaire PQ1 concerned patients who underwent general anesthesia alone or combined with epidural (30 participants). Pilot questionnaire PQ2 included patients who received only regional anesthesia (spinal, epidural, or combined spinal-epidural) (30 participants). Pilot questionnaire PQ3 concerned postoperative analgesia services in 45 of the above patients with advanced analgesic techniques such as intermittent or patient-controlled epidural analgesia (PCEA) or patient-controlled intravenous analgesia (PCA). Single ideas were phrased in each question. Most replies were based on a 4-point Likert scale, with a few in a binary (yes/no) format. Content validity, reliability (internal consistency by measuring Cronbach's $\alpha$ coefficient), and practicability (item response rate) of the questionnaires were assessed. Items with skewed values (Cronbach's $\alpha$ value $>3$ or $<0.4$ ) were excluded. There was an open-ended question at the end of the questionnaires asking patients to add features important to them. The results from the primary study were used as the basis for the formation of the three final questionnaires (depicted in the Appendix).

\section{Data collection}

In all cases, a preanesthetic visit was performed by the resident who would participate in the delivery of the anesthesia or the anesthesiologist in charge. During the preanesthetic visit, baseline characteristics were recorded in a separate leaflet: age, sex, American Society of Anesthesiologists classification, marital status, educational level, comorbidities, and prior psychiatric history. Information concerning surgical procedure, intraoperative anesthesia, and postoperative analgesia were also recorded in the same leaflet during the stay in the post-anesthesia care unit (PACU).

Questionnaires Q1 and Q2 were distributed the morning after the operation. Patients had to complete the questionnaires on their own. Based on the type of surgery, patients with expected minor or minor to moderate postoperative pain were given only questionnaires Q1 or Q2. Questionnaire Q3 was delivered to patients with expected moderate or severe postoperative pain upon removal of PCA device or epidural catheter, 3 to 5 days postoperatively. The classification of surgeries according to the expected level of postoperative pain intensity was based on previous data, suggesting that incision size and type of surgery (minor, moderate, or major) are directly related to postoperative pain. ${ }^{8,9}$ The questionnaires were anonymous in order to guarantee objective and honest answers. Only a patient admission code number had to be recorded in all leaflets so that collected information from different documents could be matched at the end.

\section{Postoperative analgesia}

Pain assessment was performed using a visual analog scale (VAS: 0-10 cm), while one of the criteria for PACU discharge was VAS score $<4$. Patients with minor pain type of surgery were prescribed paracetamol alone or combined with codeine \pm nonsteroidal anti-inflammatory drugs for postoperative analgesia. For moderate to severe pain type of surgeries, 
multimodal postoperative analgesia was applied, including primary use of opioids, local anesthetics plus paracetamol, and/or other nonsteroidal anti-inflammatory drugs. Analgesic methods such as intermittent epidural doses, PCEA, and PCA were implemented. A postoperative analgesia care team consisting of members of the anesthesiology department (both specialists and trainees) was responsible for the management of postoperative pain in patients with epidural catheters or PCA devices, performing four daily visits. Additional calls from ward nurses concerning pain control were encountered.

\section{Statistical analysis}

In all the questionnaires, inter-item, item-dimension, and interdimension correlations (Pearson's r) were assessed. Principal component analysis with varimax rotation on the correlation matrix was performed separately for each questionnaire in order to assess distinct dimensions and the extracted factors entered into multiple logistic regression models with patient satisfaction as the dependent binary variable. Statistical significance level was set at $P<0.05$. Data analysis was performed with SPSS 13.0 (IBM Corporation, Armonk, NY).

\section{Results}

Three hundred and seventy consecutive patients over a 3 -month period were originally enrolled in the study. Nine refused to fill out the questionnaires and 16 failed to complete them. Baseline characteristics of the final 345 participants in the study (125 males and 220 females) are shown in Table 1. One hundred and eight patients who underwent operations with expected minor or minor to moderate postoperative pain (minor orthopedics and gynecological operations, thyroidectomy, laparoscopic cholecystectomy, or inguinal repair) were given only questionnaire Q1 (106 patients) or Q2 (2 patients). Additional help for postoperative pain relief was encountered in only six cases. Two hundred and thirty-seven patients experienced moderate to severe pain type of surgeries (major orthopedics and gynecological operations, laparotomies, or bowel surgeries). From the 237 patients above, 176 patients received general anesthesia \pm epidural and 61 received regional anesthesia alone. From the patients undergoing general anesthesia \pm epidural, 77 (43.75\%) used PCA, 48 (27.27\%) intermittent bolus epidural, and $51(28.97 \%)$ PCEA. In patients with regional anesthesia alone, intermittent epidural bolus was used in 52 (85.25\%), while PCEA was used in nine (14.75\%).

Satisfaction rates were generally high. According to questionnaire Q1, 69.3\% of patients with general anesthesia would
Table I Baseline characteristics of the patients $(n=345)$

\begin{tabular}{|c|c|c|c|}
\hline $\begin{array}{l}\text { Clinical } \\
\text { characteristics }\end{array}$ & $\begin{array}{l}\text { General anesthesia } \pm \\
\text { epidural } \\
(n=282)\end{array}$ & $\begin{array}{l}\text { Regional } \\
\text { anesthesia } \\
(n=63)\end{array}$ & $P$-value \\
\hline Age (years) ${ }^{a}$ & $52.5 \pm 15.5$ & $61.7 \pm 13.9$ & $<0.001$ \\
\hline BMI $\left(\mathrm{kg} / \mathrm{m}^{2}\right)^{\mathrm{a}}$ & $26.5 \pm 4.8$ & $30.0 \pm 6.6$ & $<0.001$ \\
\hline \multicolumn{4}{|l|}{ Marital status (\%) } \\
\hline Single & 44 (I5.7\%) & $8(12.7 \%)$ & 0.083 \\
\hline Married & $214(75.8 \%)$ & $44(69.8 \%)$ & \\
\hline Divorced & $14(5.0 \%)$ & $4(6.3 \%)$ & \\
\hline Widowed & $10(3.6 \%)$ & $7(11.1 \%)$ & \\
\hline \multicolumn{4}{|l|}{ Educational level (\%) } \\
\hline Elementary & $153(54.2 \%)$ & $43(68.2 \%)$ & 0.104 \\
\hline Secondary & $66(23.4 \%)$ & $9(14.3 \%)$ & \\
\hline High/academic & $63(22.3 \%)$ & $\mathrm{II}(\mathrm{I7.4 \% )}$ & \\
\hline \multicolumn{4}{|c|}{ Type of surgical procedure (\%) } \\
\hline Abdominal & $83(29.4 \%)$ & $4(6.3 \%)$ & $<0.001$ \\
\hline Laparoscopic & $21(7.5 \%)$ & $0(0 \%)$ & \\
\hline Gynecologic & $73(26.0 \%)$ & $16(25.4 \%)$ & \\
\hline Orthopedic & $52(18.5 \%)$ & 31 (49.2\%) & \\
\hline Neck & $19(6.8 \%)$ & $0(0 \%)$ & \\
\hline Other & $34(12.0 \%)$ & $12(19.0 \%)$ & \\
\hline Previous surgery (\%) & $183(65 \%)$ & $10(15.9 \%)$ & $<0.001$ \\
\hline \multicolumn{4}{|l|}{ ASA classification } \\
\hline I & $120(54.5 \%)$ & 25 (39.6\%) & \\
\hline ॥ & $100(35.5 \%)$ & $32(50.8 \%)$ & \\
\hline III & $60(21.3 \%)$ & $6(9.5 \%)$ & \\
\hline IV & $2(0.7 \%)$ & $0(0 \%)$ & \\
\hline
\end{tabular}

Note: ${ }^{\mathrm{D}}$ Data are mean \pm SD.

Abbreviations: BMI, body mass index; ASA, American Society of Anesthesiologists.

definitely like to receive and $21.8 \%$ would probably like to receive the same anesthetic regimen again in the future, and $98.6 \%$ described the anesthesia procedure as good $(22.9 \%)$ or excellent (75.7\%). According to questionnaire Q2, 85.7\% of patients with regional anesthesia would like to receive the same anesthetic regimen again in the future, and $98.4 \%$ described anesthesia procedure as good (30.1\%) or excellent (68.3\%). According to questionnaire Q3, 96.3\% of patients with general anesthesia plus epidural analgesia or PCA would like to receive the same pain treatment in the future, and $96.3 \%$ described pain management as good $(31.1 \%)$ or excellent (65.2\%). Among patients with regional anesthesia, 98\% would like to receive the same pain treatment in the future and $98 \%$ described pain management as good $(25.5 \%)$ or excellent (72.5\%).

\section{General anesthesia alone or combined with epidural}

Two hundred and eighty-two patients (98 males and 184 females) were included in the study. A principal component analysis after varimax rotation isolated eight questions grouped into four dimensions (Table 2). The four-factor 
Table 2 Principal components analysis (varimax rotation) of questionnaire QI $(n=282)$

\begin{tabular}{|c|c|c|c|c|}
\hline \multirow[t]{2}{*}{ Questionnaire item } & \multicolumn{4}{|l|}{ Dimensions } \\
\hline & $\begin{array}{l}\text { Communication with } \\
\text { anesthesiologist }\end{array}$ & $\begin{array}{l}\text { Sense of cold } / \\
\text { shivering }\end{array}$ & $\begin{array}{l}\text { Pain/perception } \\
\text { of noise }\end{array}$ & Nausea \\
\hline Did you feel pain immediately after awaking from anesthesia? & & & 0.855 & \\
\hline Could you perceive noise or voices during surgery? & & & 0.854 & \\
\hline Did you have shivering immediately after awaking from anesthesia? & & 0.885 & & \\
\hline Did you feel cold immediately after awaking from anesthesia? & & 0.883 & & \\
\hline Did you have nausea immediately after awaking from anesthesia? & & & & 0.697 \\
\hline $\begin{array}{l}\text { During the preoperative evaluation visit, did you have enough } \\
\text { time to discuss your questions concerning anesthesia with the } \\
\text { anesthesiologist? }\end{array}$ & 0.821 & & & \\
\hline $\begin{array}{l}\text { During the preoperative evaluation visit, did the anesthesiologist } \\
\text { adequately address your questions? }\end{array}$ & 0.869 & & & \\
\hline $\begin{array}{l}\text { During the preoperative evaluation visit, were you given the } \\
\text { opportunity to express your opinion about the type }\end{array}$ & 0.591 & & & \\
\hline
\end{tabular}

Note: Loadings $<0.5$ are not reported in the table.

structure accounted for $68.8 \%$ of the total variance. Each dimension was named according to its constitutive items: communication with the anesthesiologist (three items), sense of cold/shivering (two items), pain and perception of noise (two items), and sense of nausea (one item).

In the multivariable analysis, satisfaction level was independently associated with age $(P=0.043)$ and communication with the anesthesiologist $(P=0.008)$. Patient's wish to receive the same anesthetic management in the future was independently associated with age $(P=0.001)$ and communication with the anesthesiologist $(P<0.001)$ and negatively associated with sense of shivering $(P=0.002)$.

Fifty-three out of the 282 patients mentioned some dissatisfaction points. Time delay in the sequence of processing (from ward to operating theater, prolonged preparation time, and from PACU until discharge to ward) was recorded in 36 patients, pain during intravenous catheterization or epidural catheters' insertion was recorded in 22 patients, and noise during preparation in the operating theater was recorded in eleven patients.

\section{Regional anesthesia}

Sixty-three patients (27 males and 36 females) were included in the study. A principal component analysis after varimax rotation isolated seven questions grouped into three dimensions (Table 3). The three-factor structure accounted for $68.9 \%$ of the total variance. Each dimension was named according to its constitutive items: communication with the anesthesiologist (three items), sense of cold/shivering (two items), and nausea and anxiety (two items). In the multivariable analysis, satisfaction level was independently associated in an inverse way with sense of shivering $(P=0.016)$.

Twelve out of the 63 patients mentioned some dissatisfaction points. Time delay in the sequence of processing

Table 3 Principal components analysis (varimax rotation) of questionnaire Q2 $(n=63)$

\begin{tabular}{lll}
\hline Questionnaire item & \begin{tabular}{l} 
Dimensions \\
\cline { 2 - 3 } Communication \\
with anesthesiologist
\end{tabular} & $\begin{array}{c}\text { Cold/shivering } \\
\text { Nausea/ } \\
\text { anxiety }\end{array}$ \\
\hline $\begin{array}{l}\text { Did you have shivering during surgery? } \\
\begin{array}{l}\text { Did you feel cold during surgery? } \\
\text { Did you have nausea during surgery? }\end{array}\end{array}$ & 0.739 \\
$\begin{array}{l}\text { Did you feel anxious during surgery? } \\
\text { During the preoperative evaluation visit, did you have enough time to } \\
\text { discuss your questions concerning anesthesia with the anesthesiologist? }\end{array}$ & 0.987 \\
$\begin{array}{l}\text { During the preoperative evaluation visit, did the anesthesiologist } \\
\text { adequately address your questions? }\end{array}$ & 0.987 \\
$\begin{array}{l}\text { During the preoperative evaluation visit, were you given the opportunity to } \\
\text { express your opinion about the type of anesthesia to be administered? }\end{array}$ & 0.604 & 0.877 \\
\hline
\end{tabular}

Note: Loadings $<0.5$ are not reported in the table. 
(from ward to operating theater, prolonged preparation time, and from PACU until discharge to ward) was recorded in five patients, pain during intravenous catheterization or epidural catheters' insertion was recorded in seven patients, and disturbing noise during performance in the operating theater was recorded in three patients.

\section{PACU}

Concerning stay in the PACU, statistical analysis from questionnaires Q1 and Q2 showed that pain and shivering were significantly inversely associated with the feeling of safety (Table 4). However, no significant association was found between this feeling of safety while in the PACU and the overall satisfaction from the anesthetic process.

\section{Ward care}

A principal component analysis after varimax rotation isolated eleven questions grouped into five dimensions (Table 5). The five-factor structure accounted for $68.3 \%$ of the total variance. Each dimension was named according to its constitutive items: anesthesiologist intervention upon symptoms (three items), pain (one item), care by the anesthesiologist/physical activity (three items), nausea/vomiting (two items), and anesthesiologist behavior (one item).

In the multivariable analysis after adjustment for anesthesia type (general/regional), satisfaction level was independently associated with anesthesiologist intervention upon symptoms $(P<0.001)$, care by the anesthesiologist/physical activity $(P=0.001)$, anesthesiologist behavior $(P=0.005)$, and negatively associated with pain $(P=0.004)$.

\section{Discussion}

This study shows that Greek surgical patients report high satisfaction with perioperative anesthesia care. Anesthesiologist-to-patient interaction during the entire perioperative period, absence of shivering during regional anesthesia, and adequate postoperative pain control in the

Table 4 Multivariable analysis of patient symptoms in the PACU and their association with the feeling of safety

\begin{tabular}{llll}
\hline Symptom & Odds ratio & 95\% confidence interval & P-value \\
\hline Pain & 0.22 & $0.10-0.47$ & $<0.001$ \\
Shivering & 0.36 & $0.18-0.72$ & 0.004 \\
Nausea & 0.89 & $0.23-3.41$ & 0.869 \\
Vomiting & 0.70 & $0.18-2.78$ & 0.617 \\
Pruritus & 0.65 & $0.12-0.81$ & 0.260 \\
Thirst & 0.67 & $0.33-1.35$ & 0.262 \\
Dizziness & 0.87 & $0.31-2.46$ & 0.790 \\
\hline
\end{tabular}

Abbreviation: PACU, postanesthesia care unit.

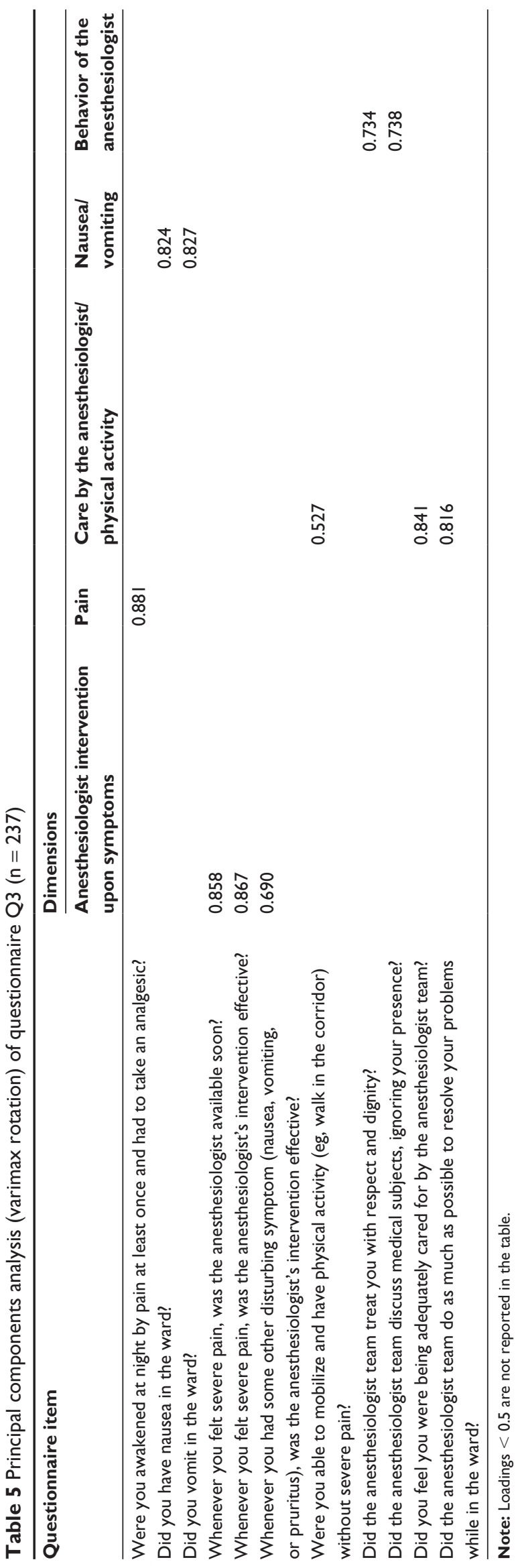


ward are also significant predictors of Greek surgical patient satisfaction. On the other hand, time delay in the sequence of processing, pain during invasive interventions, and disturbing noise perioperatively are reported as parameters of dissatisfaction.

The decision to deliver questionnaires rather than to perform interviews was based on the priority to ensure, as much as possible, anonymity, and consequently, honesty in patients' answers. ${ }^{10}$ The preoperative visit was performed by the resident or the anesthesiologist who would be responsible for the patient's intraoperative management, which ensured continuity of care. During the preanesthetic visit, information giving, relieving of patient concerns, and participation in decision making proved to be integral parts in patients' satisfaction. Interaction with the anesthesiologist during the intraoperative and immediate postoperative period was also revealed as the most important element of patient satisfaction for all patients, regardless of the type of anesthesia performed. Previous well-validated studies show results similar to ours. Heidegger et al aimed to develop a psychometric questionnaire and tested it in six hospitals in Switzerland and Austria to investigate patient satisfaction with anesthesia care. Of the most important findings were that issues such as contact with the anesthesiologist, patient informing, and involvement in deciding the anesthetic technique should be covered in a well-constructed psychometric questionnaire. ${ }^{4}$ In another study, Capuzzo et al attempted to identify items of value for surgical patients concerning satisfaction with anesthesia in an Italian hospital. They concluded that "kindness/regard of caregivers" along with "information given by the anesthetist" and "feeling safe" were good indicators for predicting patient satisfaction. They also concluded that surgical patients value emotional and relational factors most in order to be satisfied. ${ }^{3}$ Jlala et al ran a study to investigate, among other issues, patient satisfaction with perioperative care in individuals having orthopedic surgery in a university hospital. One of their conclusions was that domains such as information provision and communication between patients and health care providers should be included in a questionnaire for the assessment of patient satisfaction. Another conclusion was that patients were more satisfied if they were provided information before they underwent regional anesthesia. ${ }^{11}$

In our study, the presence of intraoperative shivering was inversely related with satisfaction in patients who underwent regional anesthesia alone. Shivering seems to be an intense symptom that strongly affects patients' emotions. This can be an important conclusion since data from regional anesthesia are limited, as Chanthong et al reported in their review of questionnaires measuring patient satisfaction in ambulatory anesthesia. ${ }^{12}$ Concerning stays in PACU, symptoms such as pain and shivering were inversely related to a feeling of safety.

Later in the ward, adequate pain control proved to be a prevalent factor in Greek patients' perception of satisfaction. In a study by Gottschalk et al, 150 adult patients who received epidural catheters for postoperative pain management were evaluated, among other issues, one week after the operation for their satisfaction with provided analgesia. The results showed a high degree of satisfaction without correlation between patients' satisfaction and recalled maximum pain levels. ${ }^{13}$ In another study by Jensen et al, data from 207 participants in the early postoperative period were used in order to examine factors that contribute to the global ratings of satisfaction with pain relief. One of the conclusions was that during the postoperative period, patients did not need complete absence of pain to be "very satisfied" with pain relief. ${ }^{14}$ We should point out that, in our study, anesthesiologists' behavior during these phases was also a significant predictor for patient satisfaction, so we can conclude that human interaction holds a leading role in all phases of perioperative care. It is in accordance with the denotation of anesthesia as "perioperative medicine" that our obligation to ensure patient's wellbeing does not end in the operating theater. It is a continuous process that expands beyond strict operation time.

In our study, delay in the sequence of processing, pain during invasive interventions, and disturbing noise perioperatively are reported as parameters of dissatisfaction. However, satisfaction rates are generally high. We were not able to show significant differences in overall patient satisfaction regarding sex, age, American Society of Anesthesiologists classification, or educational level, contrary to previous studies. Wu et al, in a 2001 review, found that advanced age and female sex consistently correlate with increased levels of satisfaction. They also noted that these results may be due to the fact that these groups receive more information or have more positive interactions with their health care providers. ${ }^{15}$ In another review, Thiedke stated: "The literature appears mixed on the importance of patients" demographic and social factors in determining satisfaction. Nevertheless the literature does shed some light on how particular demographic factors affect patient satisfaction." According to this review, the most consistent finding is the one that deals with age, since older patients seem to be more satisfied with their health care. ${ }^{16}$ She also reports that studies on the effects of biological gender on patient satisfaction are 
not consistent, with some showing that females tend to be less satisfied and others showing the opposite conclusion. She also notes that "most studies have found that individuals of lower socioeconomic status and less education tend to be less satisfied with their health care."16

One explanation for the high overall satisfaction levels observed in our study may be the continuous anesthetic care in patient management that was provided throughout the entire perioperative period, including preoperative assessment and close intraoperative and immediate postoperative care and the existence of an anesthesiologist-based analgesia care team that functioned in an organized manner and promoted enhanced communication with patients during the postoperative period. In a multicenter prospective study by Capuzzo et al, in which 1290 patients participated, factors predictive of patient satisfaction with anesthesia were investigated. They concluded that among other factors, inpatient satisfaction can be improved by enhanced postoperative visits. ${ }^{17}$ In the SIAARTI Recommendations of 2010 for acute postoperative pain treatment by Savoia et al, it is noted that "analgesia is a fundamental right of the patients" and that "treatment of postoperative pain represents a high-priority institutional objective." Among their recommendations for the improvement of an acute pain service organization, it is suggested that patient care include nights and weekends and that systematic pain assessment should be practiced. ${ }^{18}$ Most acute pain services are nurse-based, anesthesiologistsupervised. ${ }^{19,20}$ Our acute pain service is anesthesiologistbased since in our department, due to the limited number of nursing stuff, there are no nurses available to be involved. This also conforms to the institution's politics where residents have to be trained in handling acute postoperative pain and possible side effects.

Greek patient satisfaction regarding health care services has been investigated previously in a study by Papanikolaou and Ntani, who studied 367 patients with a minimum of 3 days' stay at the hospital. Reported measures included overall satisfaction, satisfaction with medical and nursing staff, satisfaction with room facilities, wait times, and extra costs. They recorded long wait times for patients to get an appointment with a physician or after examination to be admitted to the hospital. Many patients, in order to be serviced, had to rely on a personal nurse and pay extra money, and lack of staff was considered as the main drawback. However, their overall satisfaction was very high. The authors concluded that "patients are expected to act as consumers who carefully evaluate the aspects of care they receive, but this may not be the case." They also address these conclusions as "paradoxes with hospital care."21 However, in our survey, which comprised surgical patients, high satisfaction scores with anesthesia care are also followed by low recorded dissatisfaction parameters (no paradox can be addressed in our study).

Regarding the assessment of perioperative anesthesia care, one source of potential bias is that patients with ethnicities other than Greek were not included in this study. Previous studies have shown that ethnicity is an important factor for unequal levels of satisfaction among patients treated in the same health care system. ${ }^{22,23}$ Based on the fact that the purpose of the present study was to investigate, in particular, a Greek surgical population, we deliberately excluded other ethnicities. It is, however, our intention to investigate in a future study if there are any differences in perceived satisfaction with anesthesia services among patients of various ethnicities treated in the Greek health care system.

There are some limitations to our study: (1) The sample of patients who underwent regional anesthesia alone was rather small, thus reducing the safety of conclusions. (2) Our study took place in a hospital whose prevalent funding process was through public insurance. Perhaps if the study had taken place in a private institution where people would have to pay on their own or through private insurance, their demands and concerns would be greater. (3) Potential associations between patient condition or severity of disease upon discharge and patient satisfaction could not be illustrated through this study. Postdischarge contact with a supplementary questionnaire would likely have clarified whether the recorded high satisfaction rates remained unchanged with time. Noticeably, a previous study by Kaldenberg showed only weak association between patient satisfaction and health status upon discharge. ${ }^{24}$

\section{Conclusion}

Greek surgical patients report high satisfaction with perioperative anesthesia care. The present study revealed that in this population, anesthesiologist-to-patient interaction during the entire perioperative period, absence of shivering during regional anesthesia, and adequate postoperative pain control in the ward are significant predictors of patient satisfaction. On the other hand, time delay in the sequence of processing, pain during invasive interventions, and disturbing noise perioperatively are reported as parameters of dissatisfaction. However, further studies from other Greek anesthesiology departments should be performed to investigate a larger sample of this Mediterranean population. 


\section{Acknowledgments}

P Kouki and P Matsota contributed equally in the study design and motivated the study.

\section{Disclosure}

The authors report no conflicts of interest in this work. This work was not supported by any funding program.

\section{References}

1. Myles PS, Williams DL, Hendrata M, Anderson H, Weeks AM. Patient satisfaction after anaesthesia and surgery: results of a prospective survey of 10811 patients. Br J Anaesth. 2000;84(1):6-10.

2. Heidegger T, Saal D, Nuebling M. Patient satisfaction with anaesthesia care: what is patient satisfaction, how should it be measured, and what is the evidence for assuring high patient satisfaction? Best Pract Res Clin Anaesthesiol. 2006;20(2):331-346.

3. Capuzzo M, Landi F, Bassani A, Grassi L, Volta CA, Alvisi R. Emotional and interpersonal factors are most important for patient satisfaction. Acta Anaesthesiol Scand. 2005;49(6):735-742.

4. Heidegger T, Husemann Y, Nuebling M, et al. Patient satisfaction with anaesthesia care: development of a psychometric questionnaire and benchmarking among six hospitals in Switzerland and Austria. $\mathrm{Br} J$ Anaesth. 2002;89(6):863-872.

5. Auquier P, Pernoud N, Bruder N, et al. Development and validation of a perioperative satisfaction questionnaire. Anesthesiology. 2005;102(6): 1116-1123.

6. Schiff JH, Fornaschon AS, Frankenhauser S, et al. The Heidelberg Perianaesthetic Questionnaire - development of a new refined psychometric questionnaire. Anaesthesia. 2008;63(10):1096-1104.

7. Caljouw MA, Van Beuzekom M, Boer F. Patient's satisfaction with perioperative care: development, validation, and application of a questionnaire. Br J Anaesth. 2008;100(5):637-644.

8. Auburn F, Valade N, Coriat P, Riou B. Predictive factors of severe postoperative pain in the postanesthesia care unit. Anesth Analg. 2008; 106(5):1535-1541.

9. Kalkman CJ, Visser K, Moen J, Bonsel GJ, Grobbee DE, Moons KGM. Preoperative prediction of severe postoperative pain. Pain. 2003; 105(3):415-423.
10. Bauer M, Böehrer H, Aichele G, Bach A, Martin E. Measuring patient satisfaction with anaesthesia: perioperative questionnaire versus standardized face-to-face interview. Acta Anaesthesiol Scand. 2001; 45(1):65-72.

11. Jlala HA, Caljouw MA, Bedford NM, Hardman JG. Patient satisfaction with perioperative care among patients having orthopaedic surgery in a university hospital. Local Ref Anesth. 2010;3:49-55.

12. Chanthong P, Abrishami A, Wong J, Herrera F, Chung F. Systematic review of questionnaires measuring patient satisfaction in ambulatory anesthesia. Anesthesiology. 2009;110(5):1061-1067.

13. Gottschalk A, Freitag M, Liehr K, Domke A, Schuster M, Standl T. Does patient satisfaction correlate with pain level during patient-monitored epidural analgesia. Evaluation of data from postoperative pain service. Schmerz. 2004;18(2):145-150.

14. Jensen MP, Martin SA, Cheung R. The meaning of pain relief in a clinical trial. J Pain. 2005;6(6):400-406.

15. Wu CL, Naqibuddin M, Fleisher LA. Measurement of patient satisfaction as an outcome of regional anesthesia and analgesia: a systematic review. Reg Anesth Pain Med. 2001;26(3):196-208.

16. Thiedke CC. What do we really know about patient satisfaction? Fam Pract Manag. 2007;14(1):33-36.

17. Capuzzo M, Gilli G, Paparella L, et al. Factors predictive of patient satisfaction with anesthesia. Anesth Analg. 2007;105(2):435-442.

18. Savoia G, Alampi D, Amantea B, et al. Postoperative pain treatment SIAARTI Recommendations 2010 Short version. Minerva Anestesiol. 2010;76(8):657-667.

19. Shapiro A, Zohar E, Kantor M, Memrod J, Fredman B. Establishing a nurse-based, anesthesiologist-supervised inpatient acute pain service: experience of 4,617 patients. J Clin Anesth. 2004;16(6):415-420.

20. Leykin Y, Pellis T, Ambrosio C, et al. A recovery room-based acute pain service. Minerva Anestesiol. 2007;73(4):201-206.

21. Papanikolaou V, Ntani S. Addressing the paradoxes of satisfaction with hospital care. Int J Health Care Qual Assur. 2008;21(6):548-561.

22. Woods SE, Bivins R, Oteng K, Engel A. The influence of ethnicity on patient satisfaction. Ethn Health. 2005;10(3):235-242.

23. Thomas BC, Groff SL, Tsang K, Carlson LE. Patient ethnicity: a key predictor of cancer care satisfaction. Ethn Health. 2009;14(4):351-358.

24. Kaldenberg DO. Patient satisfaction and health status. Health Mark $Q$. 2001;18(3-4):81-101. 


\section{Appendix \\ Questionnaire QI}

1. Overall, how would you describe the type of anesthesia you received?

2. Could you perceive noise or voices during surgery?

3. Did you feel pain immediately after awaking from anesthesia?

4. Did you have dyspnea immediately after awaking from anesthesia?

5. Did you have shivering immediately after awaking from anesthesia?

6. Did you feel cold immediately after awaking from anesthesia?

7. Did you have nausea immediately after awaking from anesthesia?

8. Did you vomit immediately after awaking from anesthesia?

9. Did you feel that the anesthesiologists did the best they could for you perioperatively?

10. Did you feel you were being adequately cared for during your stay in the PACU?

11. Did you have other symptoms (pain, shivering, nausea, vomit, pruritus, thirst, or dizziness) during your stay in the PACU?

12. Did you have stress during your stay in the PACU?

13. Did you feel secure during your stay in the PACU?

14. Which disturbing events (noise, intense light, invasive procedures, or delay of procedures) did you experience perioperatively?c

15. During the preoperative evaluation visit, did you have enough time to discuss your questions concerning anesthesia with the anesthesiologist?

16. During the preoperative evaluation visit, did the anesthesiologist adequately address your questions?

17. During the preoperative evaluation visit, were you given the opportunity to express your opinion about the type of anesthesia to be administered?

18. Should you undergo the same operation once again, would you rather have the same anesthetic procedure?

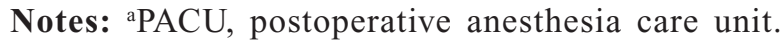
${ }^{b}$ Disturbing symptoms were offered in a list so that patients could check whatever symptom they had felt. 'Disturbing events were offered in a list so that patients could check whatever cause was a source of dissatisfaction. At the end of this question, patients had the chance to report any other causes of dissatisfaction.

\section{Questionnaire Q2}

1. Overall, how would you describe the type of anesthesia you received?

2. Did you feel pain during surgery?

3. Did you have shivering during surgery?

4. Did you feel cold during surgery?

5. Did you have nausea during surgery?

6. Did you vomit during surgery?

7. Did you feel safe during surgery?

8. Did you feel anxious during surgery?

9. Did you feel that the anesthesiologists did the best they could for you perioperatively?

10. Did you feel you were being adequately cared for during your stay in the PACU?

11. Did you have other symptoms (pain, shivering, nausea, vomiting, pruritus, thirst, or dizziness) during your stay in the PACU?

12. Did you have stress during your stay in the PACU?

13. Did you feel secure during your stay in the PACU?

14. Which disturbing events (noise, intense light, invasive procedures, or delay of procedures) did you experience perioperatively? ${ }^{\circ}$

15. During the preoperative evaluation visit, did you have enough time to discuss your questions concerning anesthesia with the anesthesiologist?

16. During the preoperative evaluation visit, did the anesthesiologist adequately address your questions?

17. During the preoperative evaluation visit, were you given the opportunity to express your opinion about the type of anesthesia to be administered?

18. Should you undergo the same operation once again, would you like to have the same anesthetic procedure?

Notes: ${ }^{\mathrm{P} A C U}$, postoperative anesthesia care unit. ${ }^{b}$ Disturbing symptoms were offered in a list so that patients could check whatever symptom they had felt. ${ }^{~}$ Disturbing events were offered in a list so that patients could check whatever cause was a source of dissatisfaction. At the end of this question, patients had the chance to report any other causes of dissatisfaction.

\section{Questionnaire Q3}

1. Overall, are you satisfied with the type of analgesia that was given to you in the ward by the anesthesiologist team?

2a. Were you awakened at night by pain at least once and had to take an analgesic?

$2 b$. If the answer in the previous question was yes, please note down approximately how many times this happened. 
3. Did you have nausea in the ward?

4. Did you vomit in the ward?

5. Did you have pruritus in the ward?

6. Whenever you felt severe pain, was the anesthesiologist available soon?

7. Whenever you felt severe pain, was the anesthesiologist's intervention effective?

8. Whenever you had some other disturbing symptom (nausea, vomiting, or pruritus), was the anesthesiologist's intervention effective?

9. Were you able to cough and move in bed without severe pain?

10. Were you able to mobilize and have physical activity (eg, walk in the corridor) without severe pain?
11. Did the anesthesiologists knock at your door before entering your room?

12. Did the anesthesiologist team discuss medical subjects, ignoring your presence?

13. Did the anesthesiologist team treat you with respect and dignity?

14. Did the anesthesiologist team provide enough information about analgesic medications and their adverse effects?

15. Did you feel you were being adequately cared for by the anesthesiologist team?

16. Did the anesthesiologist team do as much as possible to resolve your problems while in the ward?

17. Should you undergo the same operation once again, would you like to have the same anesthetic care in the ward?

\section{Publish your work in this journal}

Patient Preference and Adherence is an international, peer-reviewed, open access journal focusing on the growing importance of patient preference and adherence throughout the therapeutic continuum. Patient satisfaction, acceptability, quality of life, compliance, persistence and their role in developing new therapeutic modalities and compounds to optimize clinical outcomes for existing disease states are major areas of interest. This journal has been accepted for indexing on PubMed Central. The manuscript management system is completely online and includes a very quick and fair peer-review system. Visit http://www.dovepress.com/ testimonials.php to read real quotes from published authors. 